\title{
Commutators of intrinsic square functions on generalized Morrey spaces
}

\section{Xiaomei $\mathrm{Wu}^{1 *}$ and Taotao Zheng ${ }^{2}$}

\author{
*Correspondence: wuxm@zjnu.cn \\ 'Department of Mathematics, \\ Xingzhi College, Zhejiang Normal \\ University, Jinhua, 321004, P.R. China \\ Full list of author information is \\ available at the end of the article
}

\begin{abstract}
In this paper, we obtain the boundedness of intrinsic square functions and their commutators generated with BMO functions on generalized Morrey spaces. Our theorems extend some well-known results.
\end{abstract}

MSC: 42B20; 42B35

Keywords: intrinsic square functions; commutators; generalized Morrey spaces; BMO functions

\section{Introduction}

The intrinsic square functions were first introduced by Wilson in $[1,2]$. They are defined as follows. For $0<\alpha \leq 1$, let $\mathcal{C}_{\alpha}$ be the family of functions $\phi: \mathbb{R}^{n} \mapsto \mathbb{R}$ such that $\phi^{\prime}$ s support is contained in $\{x:|x| \leq 1\}, \int \phi d x=0$, and for $x, x^{\prime} \in \mathbb{R}^{n}$,

$$
\left|\phi(x)-\phi\left(x^{\prime}\right)\right| \leq\left|x-x^{\prime}\right|^{\alpha}
$$

For $(y, t) \in \mathbb{R}_{+}^{n+1}$ and $f \in L_{\text {loc }}^{1}\left(\mathbb{R}^{n}\right)$, set

$$
A_{\alpha} f(t, y) \equiv \sup _{\phi \in \mathcal{C}_{\alpha}}\left|f * \phi_{t}(y)\right|
$$

where $\phi_{t}(y)=t^{-n} \phi\left(\frac{y}{t}\right)$. Then we define the varying-aperture intrinsic square (intrinsic Lusin) function of $f$ by the formula

$$
G_{\alpha, \beta}(f)(x)=\left(\iint_{\Gamma_{\beta}(x)}\left(A_{\alpha} f(t, y)\right)^{2} \frac{d y d t}{t^{n+1}}\right)^{\frac{1}{2}},
$$

where $\Gamma_{\beta}(x)=\left\{(y, t) \in \mathbb{R}_{+}^{n+1}:|x-y|<\beta t\right\}$. Denote $G_{\alpha, 1}(f)=G_{\alpha}(f)$.

This function is independent of any particular kernel, such as Poisson kernel. It dominates pointwise the classical square function (Lusin area integral) and its real-variable generalizations. Although the function $G_{\alpha, \beta}(f)$ depends on the kernels with uniform compact support, there is a pointwise relation between $G_{\alpha, \beta}(f)$ with different $\beta(\beta \geq 1)$ :

$$
G_{\alpha, \beta}(f)(x) \leq \beta^{\frac{3 n}{2}+\alpha} G_{\alpha}(f)(x) .
$$

We refer for details to [1].

\section{Springer}

O2014 Wu and Zheng; licensee Springer. This is an Open Access article distributed under the terms of the Creative Commons Attribution License (http://creativecommons.org/licenses/by/2.0), which permits unrestricted use, distribution, and reproduction in any medium, provided the original work is properly cited. 
The intrinsic Littlewood-Paley $g$-function and the intrinsic $g_{\lambda}^{*}$-function are defined, respectively, by

$$
\begin{aligned}
& g_{\alpha} f(x)=\left(\int_{0}^{\infty}\left(A_{\alpha} f(t, y)\right)^{2} \frac{d t}{t}\right)^{\frac{1}{2}}, \\
& g_{\lambda, \alpha}^{*} f(x)=\left(\iint_{\mathbb{R}_{+}^{n+1}}\left(\frac{t}{t+|x-y|}\right)^{n \lambda}\left(A_{\alpha} f(t, y)\right)^{2} \frac{d y d t}{t^{n+1}}\right)^{\frac{1}{2}} .
\end{aligned}
$$

In [1], Wilson proved the following result.

Theorem A Let $1<p<\infty, 0<\alpha \leq 1$, then $G_{\alpha}$ is bounded from $L^{p}\left(\mathbb{R}^{n}\right)$ to itself.

After that, Huang and Liu [3] studied the boundedness of intrinsic square functions on weighted Hardy spaces. Moreover, they characterized the weighted Hardy spaces by intrinsic square functions. In [4] and [5], Wang and Liu obtained some weak type estimates on weighted Hardy spaces. In [6] and [7], Wang considered intrinsic functions and the commutators generated with BMO functions on weighted Morrey spaces. Let $b$ be a locally integrable function on $\mathbb{R}^{n}$. Setting

$$
A_{\alpha, b} f(t, y) \equiv \sup _{\phi \in \mathcal{C}_{\alpha}}\left|\int_{\mathbb{R}^{n}}[b(x)-b(z)] \phi_{t}(y-z) f(z) d z\right|,
$$

the commutators are defined by

$$
\begin{aligned}
& {\left[b, G_{\alpha}\right] f(x)=\left(\iint_{\Gamma(x)}\left(A_{\alpha, b} f(t, y)\right)^{2} \frac{d y d t}{t^{n+1}}\right)^{\frac{1}{2}},} \\
& {\left[b, g_{\alpha}\right] f(x)=\left(\int_{0}^{\infty}\left(A_{\alpha, b} f(t, y)\right)^{2} \frac{d t}{t}\right)^{\frac{1}{2}},}
\end{aligned}
$$

and

$$
\left[b, g_{\lambda, \alpha}^{*}\right] f(x)=\left(\iint_{\mathbb{R}_{+}^{n+1}}\left(\frac{t}{t+|x-y|}\right)^{\lambda n}\left(A_{\alpha, b} f(t, y)\right)^{2} \frac{d y d t}{t^{n+1}}\right)^{\frac{1}{2}} .
$$

A function $f \in L_{\text {loc }}^{1}\left(\mathbb{R}^{n}\right)$ is said to be in $\mathrm{BMO}\left(\mathbb{R}^{n}\right)$ if

$$
\|f\|_{*}=\sup _{x \in \mathbb{R}^{n}, r>0} \frac{1}{|B(x, r)|} \int_{B(x, r)}\left|f(y)-f_{B(x, r)}\right| d y<\infty
$$

where $f_{B(x, r)}=\frac{1}{|B(x, r)|} \int_{B(x, r)} f(y) d y$.

In this paper, we will consider $G_{\alpha}, g_{\alpha}, g_{\lambda, \alpha}^{*}$ and their commutators on generalized Morrey spaces. Let $\varphi(x, r)$ be a positive measurable function on $\mathbb{R}^{n} \times \mathbb{R}^{+}$. For any $f \in L_{\text {loc }}^{p}\left(\mathbb{R}^{n}\right)$, we denote by $L^{p, \varphi}\left(\mathbb{R}^{n}\right)$ the generalized Morrey spaces, if

$$
\|f\|_{L^{p, \varphi}\left(\mathbb{R}^{n}\right)}=\sup _{x \in \mathbb{R}^{n}, r>0} \varphi(x, r)^{-1}\left(\int_{B(x, r)}|f(x)|^{p} d x\right)^{\frac{1}{p}}<\infty .
$$


In [8], Mizuhara introduced these generalized Morrey spaces $L^{p, \varphi}\left(\mathbb{R}^{n}\right)$ and discussed the boundedness of the Calderón-Zygmund singular integral operators. Note that the generalized Morrey spaces $L^{p, \omega}\left(\mathbb{R}^{n}\right)$ with normalized norm

$$
\|f\|_{L^{p, \omega}\left(\mathbb{R}^{n}\right)}=\sup _{x \in \mathbb{R}^{n}, r>0} \omega(x, r)^{-1}|B(x, r)|^{-\frac{1}{p}}\left(\int_{B(x, r)}|f(x)|^{p} d x\right)^{\frac{1}{p}}
$$

were first defined by Guliyev in [9]. When $\omega(x, r)=r^{\frac{\lambda-n}{p}}, L^{p, \omega}\left(\mathbb{R}^{n}\right)=L^{p, \lambda}\left(\mathbb{R}^{n}\right)$. It is the classical Morrey space which was first introduced by Morrey in [10]. There are many papers discussed the conditions on $\omega(x, r)$ to obtain the boundedness of operators on the generalized Morrey spaces. For example, in [8], the function $\varphi$ is supposed to be a positively growth function and satisfy the double condition: for all $r>0, \varphi(2 r) \leq D \varphi(r)$, where $D \geq 1$ is a constant independent of $r$. This type of conditions on $\varphi$ is studied by many authors; see, for example, [11, 12]. In [13], the following statement was proved by Nakai for the Calderón-Zygmund singular integral operators $\mathrm{T}$.

Theorem B Let $1 \leq p<\infty$ and let $\omega(x, r)$ satisfy the conditions

$$
c^{-1} \omega(x, r) \leq \omega(x, t) \leq c \omega(x, r),
$$

whenever $r \leq t \leq 2 r$, where $c(\geq 1)$ does not depend on $t, r, x \in \mathbb{R}^{n}$ and

$$
\int_{r}^{\infty} \omega(x, t)^{p} \frac{d t}{t} \leq c \omega(x, r)^{p}
$$

where $c$ does not depend on $x$ and $r$. Then the operator $\mathrm{T}$ is bounded on $L^{p, \omega}\left(\mathbb{R}^{n}\right)$ for $p>1$ and from $L^{1, \omega}\left(\mathbb{R}^{n}\right)$ to $\mathrm{W} L^{1, \omega}\left(\mathbb{R}^{n}\right)$ for $p=1$.

The following statement, containing some results which were obtained in [8] and [13], was proved by Guliyev in $[14,15]$ (also see [16]).

Theorem C Let $1 \leq p<\infty$ and let the pair $\left(\omega_{1}, \omega_{2}\right)$ satisfy the condition

$$
\int_{t}^{\infty} \omega_{1}(x, r) \frac{d r}{r} \leq c \omega_{2}(x, t)
$$

where $c$ does not depend on $x$ and $t$. Then the operator $\mathrm{T}$ is bounded from $L^{p, \omega_{1}}\left(\mathbb{R}^{n}\right)$ to $L^{p, \omega_{2}}\left(\mathbb{R}^{n}\right)$ for $p>1$ and from $L^{1, \omega_{1}}\left(\mathbb{R}^{n}\right)$ to $\mathrm{W} L^{1, \omega_{2}}\left(\mathbb{R}^{n}\right)$ for $p=1$.

Recently, in [17] and [9], Guliyev et al. introduced a weaker condition for the boundedness of Calderón-Zygmund singular integral operators from $L^{p, \omega_{1}}\left(\mathbb{R}^{n}\right)$ to $L^{p, \omega_{2}}\left(\mathbb{R}^{n}\right)$ : If $1 \leq p<+\infty$, for any $x \in \mathbb{R}^{n}$ and $t>0$, there exists a constant $c>0$, such that

$$
\int_{t}^{\infty} \frac{\operatorname{essinf}_{r<s<\infty} \omega_{1}(x, s) s^{\frac{n}{p}}}{r^{\frac{n}{p}+1}} d r \leq c \omega_{2}(x, t)
$$

By an easy computation, we can check that if the pair $\left(\omega_{1}, \omega_{2}\right)$ satisfies double condition, then it will satisfy condition (1). Moreover, if $\left(\omega_{1}, \omega_{2}\right)$ satisfies condition (1), it will also 
satisfy condition (2). But the opposite is not true. We refer to [13] and Remark 4.7 in [9] for details.

In this paper, we will obtain the boundedness of the intrinsic function, the intrinsic Littlewood-Paley $g$ function, the intrinsic $g_{\lambda}^{*}$ function and their commutators on generalized Morrey spaces when the pair $\left(\omega_{1}, \omega_{2}\right)$ satisfies condition (2) or the following inequality:

$$
\int_{t}^{\infty}\left(1+\ln \frac{r}{t}\right) \frac{\operatorname{essinf}_{r<s<\infty} \omega_{1}(x, s) s^{\frac{n}{p}}}{r^{\frac{n}{p}+1}} d r \leq c \omega_{2}(x, t)
$$

Our main results in this paper are stated as follows.

Theorem 1.1 Let $1<p<\infty, 0<\alpha \leq 1$, let $\left(\omega_{1}, \omega_{2}\right)$ satisfy condition (2), then $G_{\alpha}$ is bounded from $L^{p, \omega_{1}}\left(\mathbb{R}^{n}\right)$ to $L^{p, \omega_{2}}\left(\mathbb{R}^{n}\right)$.

Theorem 1.2 Let $1<p<\infty, 0<\alpha \leq 1$, let $\left(\omega_{1}, \omega_{2}\right)$ satisfy condition (2), then for $\lambda>3+\frac{2 \alpha}{n}$, we have $g_{\lambda, \alpha}^{*}$ is bounded from $L^{p, \omega_{1}}\left(\mathbb{R}^{n}\right)$ to $L^{p, \omega_{2}}\left(\mathbb{R}^{n}\right)$.

Theorem 1.3 Let $1<p<\infty, 0<\alpha \leq 1, b \in \mathrm{BMO}$, let $\left(\omega_{1}, \omega_{2}\right)$ satisfy condition (3), then $\left[b, G_{\alpha}\right]$ is bounded from $L^{p, \omega_{1}}\left(\mathbb{R}^{n}\right)$ to $L^{p, \omega_{2}}\left(\mathbb{R}^{n}\right)$.

Theorem 1.4 Let $1<p<\infty, 0<\alpha \leq 1, b \in \mathrm{BMO}$, let $\left(\omega_{1}, \omega_{2}\right)$ satisfy condition (3), then for $\lambda>3+\frac{2 \alpha}{n},\left[b, g_{\lambda, \alpha}^{*}\right]$ is bounded from $L^{p, \omega_{1}}\left(\mathbb{R}^{n}\right)$ to $L^{p, \omega_{2}}\left(\mathbb{R}^{n}\right)$.

In [1], the author proved that the functions $G_{\alpha}$ and $g_{\alpha}$ are pointwise comparable. Thus, as a consequence of Theorem 1.1 and Theorem 1.3, we have the following results.

Corollary 1.5 Let $1<p<\infty, 0<\alpha \leq 1$, let $\left(\omega_{1}, \omega_{2}\right)$ satisfy condition (2), then $g_{\alpha}$ is bounded from $L^{p, \omega_{1}}\left(\mathbb{R}^{n}\right)$ to $L^{p, \omega_{2}}\left(\mathbb{R}^{n}\right)$.

Corollary 1.6 Let $1<p<\infty, 0<\alpha \leq 1, b \in \mathrm{BMO}$, and let ( $\left.\omega_{1}, \omega_{2}\right)$ satisfy condition (3), then $\left[b, g_{\alpha}\right]$ is bounded from $L^{p, \omega_{1}}\left(\mathbb{R}^{n}\right)$ to $L^{p, \omega_{2}}\left(\mathbb{R}^{n}\right)$.

Throughout this paper, we use the notation $A \preceq B$ to mean that there is a positive constant $C(\geq 1)$ independent of all essential variables such that $A \leq C B$. Moreover, $C$ maybe different from place to place.

\section{Proofs of main theorems}

Before proving the main theorems, we need the following lemmas.

Lemma 2.1 ([18]) The inequality ess $\sup _{t>0} \omega(t) H g(t) \preceq \operatorname{ess}_{\sup } \operatorname{s}_{t>0} v(t) g(t)$ holds for all nonnegative and non-increasing $g$ on $(0, \infty)$ if and only if

$$
A:=\sup _{t>0} \frac{\omega(t)}{t} \int_{0}^{t} \frac{d r}{\operatorname{ess} \sup _{0<s<r} v(s)}<\infty
$$

where $H g(t)$ is the Hardy operator $H g(t):=\frac{1}{t} \int_{0}^{t} g(r) d r, 0<t<\infty$. 
Lemma 2.2 ([19]) (1) For $1<p<\infty$,

$$
\|f\|_{*} \approx \sup _{x \in \mathbb{R}^{n}, r>0}\left(\frac{1}{|B(x, r)|} \int_{B(x, r)}\left|f(y)-f_{B(x, r)}\right|^{p} d y\right)^{\frac{1}{p}}
$$

(2) Let $f \in \mathrm{BMO}\left(\mathbb{R}^{n}\right), 0<2 r<t$, then

$$
\left|f_{B(x, r)}-f_{B(x, t)}\right| \preceq\|f\|_{*} \ln \frac{t}{r} .
$$

Lemma 2.3 For $j \in \mathbb{Z}^{+}$, denote

$$
G_{\alpha, 2^{j}}(f)(x)=\left(\int_{0}^{\infty} \int_{|x-y| \leq 2^{j} t}\left(A_{\alpha} f(y, t)\right)^{2} \frac{d y d t}{t^{n+1}}\right)^{\frac{1}{2}} .
$$

Let $1<p<\infty, 0<\alpha \leq 1$, then we have

$$
\left\|G_{\alpha, j^{j}}(f)\right\|_{L^{p}\left(\mathbb{R}^{n}\right)} \preceq 2^{j\left(\frac{3 n}{2}+\alpha\right)}\left\|G_{\alpha}(f)\right\|_{L^{p}\left(\mathbb{R}^{n}\right)} .
$$

From [1], we know that

$$
G_{\alpha, \beta}(f)(x) \leq \beta^{\frac{3 n}{2}+\alpha} G_{\alpha}(f)(x) .
$$

Then, by an easy computation, we get Lemma 2.3.

By a similar argument as in [20], we can easily get the following lemma.

Lemma 2.4 Let $1<p<\infty, 0<\alpha \leq 1$, then the commutators $\left[b, G_{\alpha}\right]$ is bounded from $L^{p}\left(\mathbb{R}^{n}\right)$ to itself whenever $b \in \mathrm{BMO}$.

Now we are in a position to prove the theorems.

Proof of Theorem 1.1 The main ideas of these proofs come from [9]. We decompose $f=$ $f_{1}+f_{2}$, where $f_{1}(y)=f(y) \chi_{2 B}(y), f_{2}(y)=f(y)-f_{1}(y), B:=B\left(x_{0}, r\right)$. Then

$$
\left\|G_{\alpha} f\right\|_{L^{p}\left(B\left(x_{0}, r\right)\right)} \leq\left\|G_{\alpha} f_{1}\right\|_{L^{p}\left(B\left(x_{0}, r\right)\right)}+\left\|G_{\alpha} f_{2}\right\|_{L^{p}\left(B\left(x_{0}, r\right)\right)}:=I+I I .
$$

First, let us estimate I. By Theorem A, we obtain

$$
I \leq\left\|G_{\alpha} f_{1}\right\|_{L^{p}\left(\mathbb{R}^{n}\right)} \preceq\left\|f_{1}\right\|_{L^{p}\left(\mathbb{R}^{n}\right)}=\|f\|_{L^{p}(2 B)} \preceq r^{\frac{n}{p}} \int_{2 r}^{\infty}\|f\|_{L^{p}\left(B\left(x_{0}, t\right)\right)} t^{-\frac{n}{p}-1} d t .
$$

Then let us estimate II. Recalling the properties of function $\phi$, we know that

$$
\left|f_{2} * \phi_{t}(y)\right|=\left|t^{-n} \int_{|y-z| \leq t} \phi\left(\frac{y-z}{t}\right) f_{2}(z) d z\right| \preceq t^{-n} \int_{|y-z| \leq t}\left|f_{2}(z)\right| d z .
$$

Since $x \in B\left(x_{0}, r\right),(y, t) \in \Gamma(x)$ and $\left|z-x_{0}\right| \geq 2 r$, we have

$$
r \leq\left|z-x_{0}\right|-\left|x_{0}-x\right| \leq|x-z| \leq|x-y|+|y-z| \leq 2 t .
$$


So, we obtain

$$
\begin{aligned}
G_{\alpha} f_{2}(x) & \leq\left(\iint_{\Gamma(x)}\left|t^{-n} \int_{|y-z| \leq t}\right| f_{2}(z)|d z|^{2} \frac{d y d t}{t^{n+1}}\right)^{\frac{1}{2}} \\
& \leq\left(\int_{t>r / 2} \int_{|x-y|<t}\left(\int_{|z-x| \leq 2 t}\left|f_{2}(z)\right| d z\right)^{2} \frac{d y d t}{t^{3 n+1}}\right)^{\frac{1}{2}} \\
& \preceq\left(\int_{t>r / 2}\left(\int_{|z-x| \leq 2 t}\left|f_{2}(z)\right| d z\right)^{2} \frac{d t}{t^{2 n+1}}\right)^{\frac{1}{2}} .
\end{aligned}
$$

By Minkowski's inequality and $|z-x| \geq\left|z-x_{0}\right|-\left|x_{0}-x\right| \geq \frac{1}{2}\left|z-x_{0}\right|$, we have

$$
\begin{aligned}
G_{\alpha} f_{2}(x) & \preceq \int_{\mathbb{R}^{n}}\left(\int_{t>\frac{|z-x|}{2}} \frac{d t}{t^{2 n+1}}\right)^{\frac{1}{2}}\left|f_{2}(z)\right| d z \\
& \preceq \int_{\left|z-x_{0}\right|>2 r} \frac{|f(z)|}{|z-x|^{n}} d z \preceq \int_{\left|z-x_{0}\right|>2 r} \frac{|f(z)|}{\left|z-x_{0}\right|^{n}} d z \\
& \preceq \int_{\left|z-x_{0}\right|>2 r}|f(z)| \int_{\left|z-x_{0}\right|}^{+\infty} \frac{1}{t^{n+1}} d t d z \\
& =\int_{2 r}^{+\infty} \int_{2 r<\left|z-x_{0}\right|<t}|f(z)| d z \frac{1}{t^{n+1}} d t \preceq \int_{2 r}^{\infty}\|f\|_{L^{p}\left(B\left(x_{0}, t\right)\right)} t^{-\frac{n}{p}-1} d t .
\end{aligned}
$$

The last inequality is due to Hölder's inequality. Thus,

$$
\left\|G_{\alpha} f_{2}\right\|_{L^{p}\left(B\left(x_{0}, r\right)\right)} \preceq r^{\frac{n}{p}} \int_{2 r}^{\infty}\|f\|_{L^{p}\left(B\left(x_{0}, t\right)\right)} t^{-\frac{n}{p}-1} d t .
$$

By combining (5) and (6), we have

$$
\left\|G_{\alpha} f\right\|_{L^{p}\left(B\left(x_{0}, r\right)\right)} \preceq r^{\frac{n}{p}} \int_{2 r}^{\infty}\|f\|_{L^{p}\left(B\left(x_{0}, t\right)\right)} t^{-\frac{n}{p}-1} d t .
$$

So, let $t=s^{-\frac{p}{n}}$; we have

$$
\begin{aligned}
& \left\|G_{\alpha} f\right\|_{L^{p, \omega_{2}}\left(\mathbb{R}^{n}\right)} \preceq \sup _{x_{0} \in \mathbb{R}^{n}, r>0} \omega_{2}\left(x_{0}, r\right)^{-1}\left|B\left(x_{0}, r\right)\right|^{-\frac{1}{p}} r^{\frac{n}{p}} \int_{2 r}^{\infty}\|f\|_{L^{p}\left(B\left(x_{0}, t\right)\right)} \frac{1}{t^{\frac{n}{p}+1}} d t \\
& \preceq \sup _{x_{0} \in \mathbb{R}^{n}, r>0} \omega_{2}\left(x_{0}, r\right)^{-1} \int_{0}^{r^{-\frac{n}{p}}}\|f\|_{L^{p}\left(B\left(x_{0}, s^{-\frac{p}{n}}\right)\right)} d s \\
& =\sup _{x_{0} \in \mathbb{R}^{n}, r>0} \omega_{2}\left(x_{0}, r^{-\frac{p}{n}}\right)^{-1} \int_{0}^{r}\|f\|_{L^{p}\left(B\left(x_{0}, s^{-\frac{p}{n}}\right)\right)} d s .
\end{aligned}
$$

Take $w(t)=\omega_{2}\left(x_{0}, t^{-\frac{p}{n}}\right)^{-1} t, v(t)=\omega_{1}\left(x_{0}, t^{-\frac{p}{n}}\right)^{-1} t$. Since $\left(\omega_{1}, \omega_{2}\right)$ satisfies condition (2), we can verify that $w(t), v(t)$ satisfy condition (4). Let $g(s)=\|f\|_{L^{p}\left(B\left(x_{0}, s^{\left.\left.-\frac{p}{n}\right)\right)}\right.\right.}$. Obviously, it is decreasing on variable $s$. So, by Lemma 2.1, we can conclude the following estimates:

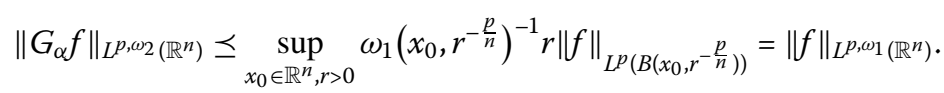


Proof of Theorem 1.2

$$
\begin{aligned}
{\left[g_{\lambda, \alpha}^{*}(f)(x)\right]^{2}=} & \int_{0}^{\infty} \int_{|x-y|<t}\left(\frac{t}{t+|x-y|}\right)^{n \lambda}\left(A_{\alpha} f(y, t)\right)^{2} \frac{d y d t}{t^{n+1}} \\
& +\int_{0}^{\infty} \int_{|x-y| \geq t}\left(\frac{t}{t+|x-y|}\right)^{n \lambda}\left(A_{\alpha} f(y, t)\right)^{2} \frac{d y d t}{t^{n+1}} \\
:= & I I I+I V .
\end{aligned}
$$

First, let us estimate III:

$$
I I I \leq \int_{0}^{+\infty} \int_{|x-y|<t}\left(A_{\alpha} f(y, t)\right)^{2} \frac{d y d t}{t^{n+1}}=\left(G_{\alpha} f(x)\right)^{2} .
$$

Then let us estimate IV:

$$
\begin{aligned}
I V & \leq \sum_{j=1}^{\infty} \int_{0}^{\infty} \int_{2^{j-1} t \leq|x-y| \leq 2 j t}\left(\frac{t}{t+|x-y|}\right)^{n \lambda}\left(A_{\alpha} f(y, t)\right)^{2} \frac{d y d t}{t^{n+1}} \\
& \leq \sum_{j=1}^{\infty} \int_{0}^{\infty} \int_{2^{j-1} t \leq|x-y| \leq 2 j t} 2^{-j n \lambda}\left(A_{\alpha} f(y, t)\right)^{2} \frac{d y d t}{t^{n+1}} \\
& \leq \sum_{j=1}^{\infty} 2^{-j n \lambda} \int_{0}^{\infty} \int_{|x-y| \leq 2^{j} t}\left(A_{\alpha} f(y, t)\right)^{2} \frac{d y d t}{t^{n+1}} \\
& :=\sum_{j=1}^{\infty} 2^{-j n \lambda}\left(G_{\alpha, 2 j}(f)(x)\right)^{2} .
\end{aligned}
$$

Thus,

$$
\left\|g_{\lambda, \alpha}^{*}(f)\right\|_{L^{p, \omega_{2}\left(\mathbb{R}^{n}\right)}} \leq\left\|G_{\alpha} f\right\|_{L^{p, \omega_{2}\left(\mathbb{R}^{n}\right)}}+\sum_{j=1}^{\infty} 2^{-\frac{j n \lambda}{2}}\left\|G_{\alpha, 2 j}(f)\right\|_{L^{p, \omega_{2}\left(\mathbb{R}^{n}\right)}} .
$$

By Theorem 1.1, we have

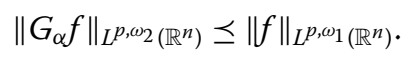

To complete the proof, it suffices to estimate $\left\|G_{\alpha, 2 j}(f)\right\|_{L^{p, \omega_{2}}\left(\mathbb{R}^{n}\right)}$. Take $f_{1}(y)=f(y) \chi_{2 B}(y)$, $f_{2}(y)=f(y)-f_{1}(y), 2 B=B\left(x_{0}, 2 r\right)$. Then

$$
\left\|G_{\alpha, j}(f)\right\|_{L^{p}\left(B\left(x_{0}, r\right)\right)} \leq\left\|G_{\alpha, j}\left(f_{1}\right)\right\|_{L^{p}\left(B\left(x_{0}, r\right)\right)}+\left\|G_{\alpha, j}\left(f_{2}\right)\right\|_{L^{p}\left(B\left(x_{0}, r\right)\right)^{\prime}}
$$

For the first part, by Lemma 2.3, we obtain

$$
\begin{aligned}
\left\|G_{\alpha, j}\left(f_{1}\right)\right\|_{L^{p}\left(B\left(x_{0}, r\right)\right)} & \preceq 2^{j\left(\frac{3 n}{2}+\alpha\right)}\left\|G_{\alpha}\left(f_{1}\right)\right\|_{L^{p}\left(\mathbb{R}^{n}\right)} \preceq 2^{j\left(\frac{3 n}{2}+\alpha\right)}\|f\|_{L^{p}(2 B)} \\
& \preceq 2^{j\left(\frac{3 n}{2}+\alpha\right)} r^{\frac{n}{p}} \int_{2 r}^{\infty}\|f\|_{L^{p}\left(B\left(x_{0}, t\right)\right)} \frac{1}{t^{\frac{n}{p}+1}} d t .
\end{aligned}
$$


For the other part, we know

$$
\begin{aligned}
G_{\alpha, 2^{j}}\left(f_{2}\right)(x) & =\left(\int_{0}^{\infty} \int_{|x-y| \leq 2^{j} t}\left(A_{\alpha} f_{2}(y, t)\right)^{2} \frac{d y d t}{t^{n+1}}\right)^{\frac{1}{2}} \\
& =\left(\int_{0}^{\infty} \int_{|x-y| \leq \nu^{j} t}\left(\sup _{\phi \in \mathcal{C}_{\alpha}}\left|f_{2} * \phi_{t}(y)\right|\right)^{2} \frac{d y d t}{t^{n+1}}\right)^{\frac{1}{2}} \\
& \preceq\left(\int_{0}^{\infty} \int_{|x-y| \leq \nu^{j} t}\left(\int_{|z-y| \leq t}\left|f_{2}(z)\right| d z\right)^{2} \frac{d y d t}{t^{3 n+1}}\right)^{\frac{1}{2}} .
\end{aligned}
$$

Since $|z-x| \leq|z-y|+|y-x| \leq 2^{j+1} t$, by Minkowski's inequality, we get

$$
\begin{aligned}
G_{\alpha, 2 j}\left(f_{2}\right)(x) & \preceq\left(\int_{0}^{\infty} \int_{|x-y| \leq 2 j t}\left(\int_{|z-x| \leq 2^{j+1} t}\left|f_{2}(z)\right| d z\right)^{2} \frac{d y d t}{t^{3 n+1}}\right)^{\frac{1}{2}} \\
& \preceq\left(\int_{0}^{\infty}\left(\int_{|z-x| \leq 2^{j+1} t}\left|f_{2}(z)\right| d z\right)^{2} \frac{2^{j n} d t}{t^{2 n+1}}\right)^{\frac{1}{2}} \\
& \leq 2^{\frac{j n}{2}} \int_{\mathbb{R}^{n}}\left(\int_{t \geq \frac{|z-x|}{2^{j+1}}}\left|f_{2}(z)\right|^{2} \frac{1}{t^{2 n+1}} d t\right)^{\frac{1}{2}} d z \\
& \leq 2^{\frac{3 j n}{2}} \int_{\left|z-x_{0}\right|>2 r} \frac{|f(z)|}{|z-x|^{n}} d z .
\end{aligned}
$$

For $x \in B\left(x_{0}, r\right)$, we have $|z-x| \geq\left|z-x_{0}\right|-\left|x_{0}-x\right| \geq\left|z-x_{0}\right|-\frac{1}{2}\left|z-x_{0}\right|=\frac{1}{2}\left|z-x_{0}\right|$. So by Fubini's theorem and Hölder's inequality, we obtain

$$
\begin{aligned}
G_{\alpha, 2^{j}}\left(f_{2}\right)(x) & \preceq 2^{\frac{3 j n}{2}} \int_{\left|z-x_{0}\right|>2 r} \frac{|f(z)|}{\left|z-x_{0}\right|^{n}} d z \\
& \preceq 2^{\frac{3 j n}{2}} \int_{\left|z-x_{0}\right|>2 r}|f(z)| \int_{\left|z-x_{0}\right|}^{\infty} \frac{1}{t^{n+1}} d t d z \\
& =2^{\frac{3 j n}{2}} \int_{2 r}^{\infty} \int_{\left|z-x_{0}\right|<t}|f(z)| \frac{1}{t^{n+1}} d z d t \\
& \leq 2^{\frac{3 j n}{2}} \int_{2 r}^{\infty}\|f\|_{L^{p}\left(B\left(x_{0}, t\right)\right)} \frac{1}{t^{\frac{n}{p}+1}} d t .
\end{aligned}
$$

Thus,

$$
\left\|G_{\alpha, 2 j}\left(f_{2}\right)\right\|_{L^{p}\left(B\left(x_{0}, r\right)\right)} \preceq 2^{\frac{3 j n}{2}} r^{\frac{n}{p}} \int_{2 r}^{\infty}\|f\|_{L^{p}\left(B\left(x_{0}, t\right)\right)} \frac{1}{t^{\frac{n}{p}+1}} d t .
$$

Combining by (9), (10), and (11), we have

$$
\left\|G_{\alpha, 2 j}(f)\right\|_{L^{p}\left(B\left(x_{0}, r\right)\right)} \preceq 2^{j\left(\frac{3 n}{2}+\alpha\right)} r^{\frac{n}{p}} \int_{2 r}^{\infty}\|f\|_{L^{p}\left(B\left(x_{0}, t\right)\right)} \frac{1}{t^{\frac{n}{p}+1}} d t .
$$


Wu and Zheng Journal of Inequalities and Applications 2014, 2014:128

Page 9 of 12

Thus, by substitution of variables and Lemma 2.1, we get

$$
\begin{aligned}
\left\|G_{\alpha, 2^{j}}(f)\right\|_{L^{p, \omega_{2}\left(\mathbb{R}^{n}\right)}} & \preceq 2^{j\left(\frac{3 n}{2}+\alpha\right)} \sup _{x_{0} \in \mathbb{R}^{n}, r>0} \omega_{2}\left(B\left(x_{0}, r\right)\right)^{-1}\left|B\left(x_{0}, r\right)\right|^{-\frac{1}{p}} \int_{0}^{r^{-\frac{n}{p}}}\|f\|_{L^{p}\left(B\left(x_{0}, s^{-\frac{p}{n}}\right)\right)} d s \\
& =2^{j\left(\frac{3 n}{2}+\alpha\right)} \sup _{x_{0} \in \mathbb{R}^{n}, r>0} \omega_{2}\left(x_{0}, r^{-\frac{p}{n}}\right)^{-1} \int_{0}^{r}\|f\|_{L^{p}\left(B\left(x_{0}, s^{-\frac{p}{n}}\right)\right)} d s \\
& \preceq 2^{j\left(\frac{3 n}{2}+\alpha\right)} \sup _{x_{0} \in \mathbb{R}^{n}, r>0} \omega_{1}\left(x_{0}, r^{-\frac{p}{n}}\right)^{-1} r\|f\|_{L^{p}\left(B\left(x_{0}, r^{-\frac{p}{n}}\right)\right)} \\
& =2^{j\left(\frac{3 n}{2}+\alpha\right)}\|f\|_{L^{p, \omega_{1}}\left(\mathbb{R}^{n}\right) .}
\end{aligned}
$$

Since $\lambda>3+\frac{2 \alpha}{n}$, by (7), (8) and (12), we have the desired theorem.

Proof of Theorem 1.3 We decompose $f=f_{1}+f_{2}$ as in the proof of Theorem 1.2, where $f_{1}=f \chi_{2 B}$ and $f_{2}=f-f_{1}$. Then

$$
\left\|\left[b, G_{\alpha}\right] f\right\|_{L^{p}\left(B\left(x_{0}, r\right)\right)} \leq\left\|\left[b, G_{\alpha}\right] f_{1}\right\|_{L^{p}\left(B\left(x_{0}, r\right)\right)}+\left\|\left[b, G_{\alpha}\right] f_{2}\right\|_{L^{p}\left(B\left(x_{0}, r\right)\right)} .
$$

By Lemma 2.4, we have

$$
\left\|\left[b, G_{\alpha}\right] f_{1}\right\|_{L^{p}\left(B\left(x_{0}, r\right)\right)} \preceq\left\|f_{1}\right\|_{L^{p}\left(\mathbb{R}^{n}\right)}=\|f\|_{L^{p}(2 B)} \preceq r^{\frac{n}{p}} \int_{2 r}^{\infty}\|f\|_{L^{p}\left(B\left(x_{0}, t\right)\right)} \frac{1}{t^{\frac{n}{p}+1}} d t .
$$

Next, we estimate the second part. We divide it into two parts. We have

$$
\begin{aligned}
& {\left[b, G_{\alpha}\right] f_{2}(x)=\left(\iint_{\Gamma(x)} \sup _{\phi \in \mathcal{C}_{\alpha}}\left|\int_{\mathbb{R}^{n}}[b(x)-b(z)] \phi_{t}(y-z) f_{2}(z) d z\right|^{2} \frac{d y d t}{t^{n+1}}\right)^{\frac{1}{2}}} \\
& \leq\left(\iint_{\Gamma(x)} \sup _{\phi \in \mathcal{C}_{\alpha}}\left|\int_{\mathbb{R}^{n}}\left[b(x)-b_{B}\right] \phi_{t}(y-z) f_{2}(z) d z\right|^{2} \frac{d y d t}{t^{n+1}}\right)^{\frac{1}{2}} \\
& +\left(\iint_{\Gamma(x)} \sup _{\phi \in \mathcal{C}_{\alpha}}\left|\int_{\mathbb{R}^{n}}\left[b_{B}-b(z)\right] \phi_{t}(y-z) f_{2}(z) d z\right|^{2} \frac{d y d t}{t^{n+1}}\right)^{\frac{1}{2}} \\
& :=V+V I \text {. }
\end{aligned}
$$

First, for V, we find that

$$
V=\left|b(x)-b_{B}\right|\left(\iint_{\Gamma(x)} \sup _{\phi \in \mathcal{C}_{\alpha}}\left|\int_{\mathbb{R}^{n}} \phi_{t}(y-z) f_{2}(z) d z\right|^{2} \frac{d y d t}{t^{n+1}}\right)^{\frac{1}{2}}=\left|b(x)-b_{B}\right| G_{\alpha} f_{2}(x) .
$$

Following the proof in Theorem 1.1, we get

$$
\begin{aligned}
& \left(\int_{B\left(x_{0}, r\right)}\left|b(x)-b_{B}\right|^{p}\left|G_{\alpha} f_{2}(x)\right|^{p} d x\right)^{\frac{1}{p}} \\
& \quad \preceq\left(\int_{B\left(x_{0}, r\right)}\left|b(x)-b_{B}\right|^{p} d x\right)^{\frac{1}{p}} \int_{2 r}^{+\infty}\|f\|_{L^{p}\left(B\left(x_{0}, t\right)\right)} \frac{d t}{t^{\frac{n}{p}+1}} \\
& \quad \preceq\|b\|_{*} r^{\frac{n}{p}} \int_{2 r}^{+\infty}\|f\|_{L^{p}\left(B\left(x_{0}, t\right)\right)} \frac{d t}{t^{\frac{n}{p}+1}} .
\end{aligned}
$$


For VI, since $|y-x|<t$, we get $|x-z|<2 t$. Thus, by Minkowski's inequality, we obtain

$$
\begin{aligned}
V I & \preceq\left(\iint_{\Gamma(x)}\left|\int_{|x-z|<2 t}\right| b_{B}-b(z)|| f_{2}(z)|d z|^{2} \frac{d y d t}{t^{3 n+1}}\right)^{\frac{1}{2}} \\
& \preceq\left(\int_{0}^{\infty}\left|\int_{|x-z|<2 t}\right| b_{B}-b(z)|| f_{2}(z)|d z|^{2} \frac{d t}{t^{2 n+1}}\right)^{\frac{1}{2}} \\
& \preceq \int_{\left|x_{0}-z\right|>2 r}\left|b_{B}-b(z)\right||f(z)| \frac{1}{|x-z|^{n}} d z .
\end{aligned}
$$

Since $|z-x| \geq \frac{1}{2}\left|z-x_{0}\right|$, by Fubini's theorem, we get

$$
\begin{aligned}
& \left(\int_{B\left(x_{0}, r\right)}|V I|^{p} d x\right)^{\frac{1}{p}} \preceq\left(\int_{B\left(x_{0}, r\right)}\left|\int_{\left|x_{0}-z\right|>2 r}\right| b_{B}-b(z)|| f(z)\left|\frac{1}{|x-z|^{n}} d z\right|^{p} d x\right)^{\frac{1}{p}} \\
& \preceq r^{\frac{n}{p}} \int_{\left|x_{0}-z\right|>2 r}\left|b_{B}-b(z)\right||f(z)| \frac{1}{\left|x_{0}-z\right|^{n}} d z \\
& \preceq r^{\frac{n}{p}} \int_{\left|x_{0}-z\right|>2 r}\left|b_{B}-b(z)\right||f(z)| \int_{\left|x_{0}-z\right|}^{+\infty} \frac{1}{t^{n+1}} d t d z \\
& \leq r^{\frac{n}{p}} \int_{2 r}^{+\infty} \int_{B\left(x_{0}, t\right)}\left|b_{B}-b(z)\right||f(z)| d z \frac{1}{t^{n+1}} d t \\
& \leq r^{\frac{n}{p}} \int_{2 r}^{+\infty} \int_{B\left(x_{0}, t\right)}\left|b_{B}-b_{B\left(x_{0}, t\right)}\right||f(z)| d z \frac{1}{t^{n+1}} d t \\
& +r^{\frac{n}{p}} \int_{2 r}^{+\infty} \int_{B\left(x_{0}, t\right)}\left|b(z)-b_{B\left(x_{0}, t\right)}\right||f(z)| d z \frac{1}{t^{n+1}} d t \\
& :=A+B \text {. }
\end{aligned}
$$

For $A$, using Lemma 2.2 and Hölder's inequality, we have

$$
\begin{aligned}
A & \preceq\|b\|_{*} r^{\frac{n}{p}} \int_{2 r}^{+\infty} \int_{B\left(x_{0}, t\right)}|f(z)| d z \frac{1}{t^{n+1}} \ln \frac{t}{r} d t \\
& \preceq r^{\frac{n}{p}} \int_{2 r}^{+\infty} \ln \frac{t}{r}\|f\|_{L^{p}\left(B\left(x_{0}, t\right)\right)} \frac{d t}{t^{\frac{n}{p}+1}} .
\end{aligned}
$$

For $B$, we denote $D=\int_{B\left(x_{0}, t\right)}|f(z)|\left|b_{B\left(x_{0}, t\right)}-b(z)\right| d z$. Then, by Hölder's inequality and Lemma 2.2, we get

$$
\begin{aligned}
D & \leq\left(\int_{B\left(x_{0}, t\right)}|f(z)|^{p} d z\right)^{\frac{1}{p}}\left(\int_{B\left(x_{0}, t\right)}\left|b_{B\left(x_{0}, t\right)}-b(z)\right|^{p^{\prime}} d z\right)^{\frac{1}{p^{\prime}}} \\
& \preceq t^{\frac{n}{p^{\prime}}}\|b\|_{*}\|f\|_{L^{p}\left(B\left(x_{0}, t\right)\right)} .
\end{aligned}
$$

This yields $B \preceq r^{\frac{n}{p}} \int_{2 r}^{+\infty}\|f\|_{L^{p}\left(B\left(x_{0}, t\right)\right)} \frac{d t}{t^{\frac{n}{p}+1}}$. Thus,

$$
\left\|\left[b, G_{\alpha}\right] f\right\|_{L^{p}\left(B\left(x_{0}, r\right)\right)} \preceq r^{\frac{n}{p}} \int_{2 r}^{\infty}\|f\|_{L^{p}\left(B\left(x_{0}, t\right)\right)} \frac{1}{t^{\frac{n}{p}+1}}\left(1+\ln \frac{t}{r}\right) d t .
$$


By a change of variables, we obtain

$$
\begin{aligned}
& \left\|\left[b, G_{\alpha}\right] f\right\|_{L^{p, \omega_{2}\left(\mathbb{R}^{n}\right)}} \\
& \preceq \sup _{x_{0} \in \mathbb{R}^{n}, r>0} \omega_{2}\left(x_{0}, r\right)^{-1}\left|B\left(x_{0}, r\right)\right|^{-\frac{1}{p}} r^{\frac{n}{p}} \int_{2 r}^{\infty}\|f\|_{L^{p}\left(B\left(x_{0}, t\right)\right)} \frac{1}{t^{\frac{n}{p}+1}}\left(1+\ln \frac{t}{r}\right) d t \\
& \preceq \sup _{x_{0} \in \mathbb{R}^{n}, r>0} \omega_{2}\left(x_{0}, r\right)^{-1} \int_{0}^{r^{-\frac{n}{p}}}\|f\|_{L^{p}\left(B\left(x_{0}, s^{-\frac{p}{n}}\right)\right)}\left(1+\ln \frac{s^{-\frac{p}{n}}}{r}\right) d s \\
& =\sup _{x_{0} \in \mathbb{R}^{n}, r>0} \omega_{2}\left(x_{0}, r^{-\frac{p}{n}}\right)^{-1} \int_{0}^{r}\|f\|_{L^{p}\left(B\left(x_{0}, s^{-\frac{p}{n}}\right)\right)}\left(1+\frac{p}{n} \ln \frac{r}{s}\right) d s .
\end{aligned}
$$

Let $w(t)=\omega_{2}\left(x_{0}, t^{-\frac{p}{n}}\right)^{-1} t, v(t)=\omega_{1}\left(x_{0}, t^{-\frac{p}{n}}\right)^{-1} t$. Since $\left(\omega_{1}, \omega_{2}\right)$ satisfies condition (3), by a similarly argument with Theorem 1.1 , we conclude the following estimates:

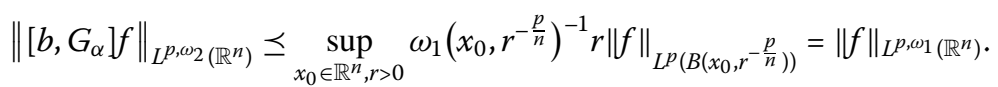

Using an argument similar to the above proofs and that of Theorem 1.2, we can also show the boundedness of $\left[b, g_{\lambda, \alpha}^{*}\right]$.

\section{Competing interests}

The author declares that they have no competing interests.

\section{Authors' contributions}

All authors read and approved the final manuscript.

\section{Author details}

${ }^{1}$ Department of Mathematics, Xingzhi College, Zhejiang Normal University, Jinhua, 321004, P.R. China. ${ }^{2}$ Department of Mathematics, Zhejiang University, Hangzhou, 310027, P.R. China.

\section{Acknowledgements}

This work was completed with the support of Scientific Research Fund of Zhejiang Provincial Education Department No. Y201225707.

Received: 25 September 2013 Accepted: 13 March 2014 Published: 28 Mar 2014

\section{References}

1. Wilson, M: The intrinsic square function. Rev. Mat. Iberoam. 23, 771-791 (2007)

2. Wilson, M: Weighted Littlewood-Paley Theory and Exponential-Square Integrability. Lecture Notes in Math., vol. 1924. Springer, Berlin (2007)

3. Huang, JZ, Liu, Y: Some characterizations of weighted Hardy spaces. J. Math. Anal. Appl. 363, 121-127 (2010)

4. Wang, H: Boundedness of intrinsic square functions on the weighted weak Hardy spaces. Integr. Equ. Oper. Theory 75, 135-149 (2013)

5. Wang, H, Liu, HP: Weak type estimates of intrinsic square functions on the weighted Hardy spaces. Arch. Math. 97, 49-59 (2011)

6. Wang, $\mathrm{H}$ : Weak type estimates for intrinsic square functions on weighted Morrey spaces. Anal. Theory Appl. 29(2), 104-119 (2013)

7. Wang, H: Intrinsic square functions on the weighted Morrey spaces. J. Math. Anal. Appl. 396, 302-314 (2012)

8. Mizuhara, T: Boundedness of some classical operators on generalized Morrey spaces. In: Lgari, S (ed.) Harmonic Analysis. ICM-90 Conference Proceedings, pp. 183-189. Springer, Tokyo (1991)

9. Guliyev, VS, Aliyev, SS, Karaman, T, Shukurov, PS: Boundedness of sublinear operators and commutators on generalized Morrey spaces. Integr. Equ. Oper. Theory 71, 327-355 (2011)

10. Morrey, C: On the solutions of quasi-linear elliptic partial differential equations. Trans. Amer. Math. Soc. 43, 126-166 (1938)

11. Ding, Y, Yang, DC, Zhou, Z: Boundedness of sublinear operators and commutators on $L^{p}\left(\mathbb{R}^{n}\right)$. Yokohama Math. J. 46, 15-27 (1998)

12. Wang, $\mathrm{H}$ : Boundedness of intrinsic square functions on generalized Morrey spaces. arXiv: $1103.1715 \mathrm{~V} 2$

13. Nakai, E: Hardy-Littlewood maximal operator, singular integral operators and Riesz potentials on generalized Morrey spaces. Math. Nachr. 166, 95-103 (1994) 
14. Guliyev, VS: Boundedness of the maximal, potential and singular operators in the generalized Morrey spaces. J. Inequal. Appl. 2009, Article ID 503948 (2009)

15. Guliyev, VS: Integral operators on function spaces on the homogeneous groups and on domains in Rn, Doctor's degree dissertation, Mat. Inst. Steklov, Moscow, 329 pp. (1994) (in Russian)

16. Guliyev, VS: Function spaces, Integral Operators and Two Weighted Inequalities on Homogeneous Groups. Some Applications, Cashioglu, Baku, 332 pp. (1999) (in Russian)

17. Aliyev, SS, Guliyev, VS: Boundedness of the parametric Marcinkiewicz integral operator and its commutators on generalized Morrey spaces. Georgian Math. J. 19, 195-208 (2012)

18. Carro, M, Pick, L, Soria, J, Stepanow, VD: On embeddings between classical Lorentz spaces. Math. Inequal. Appl. 4(3), 397-428 (2001)

19. John, F, Nirenberg, L: On functions of bounded mean oscillation. Commun. Pure Appl. Math. 14, 415-426 (1961)

20. Ding, Y, Lu, SZ, Yabuta, K: On commutators of Marcinkiewicz integrals with rough kernel. J. Math. Anal. Appl. 275, 60-68 (2002)

10.1186/1029-242X-2014-128

Cite this article as: Wu and Zheng: Commutators of intrinsic square functions on generalized Morrey spaces. Journal of Inequalities and Applications 2014, 2014:128

\section{Submit your manuscript to a SpringerOpen ${ }^{\circ}$ journal and benefit from:}

- Convenient online submission

- Rigorous peer review

Immediate publication on acceptance

Open access: articles freely available online

- High visibility within the field

- Retaining the copyright to your article 